\title{
Effect of the sun on visible clinical signs of aging in Caucasian skin
}

\author{
Frederic Flament ${ }^{1}$ \\ Roland Bazin² \\ Sabine Laquieze ${ }^{3}$ \\ Virginie Rubert ${ }^{1}$ \\ Elisa Simonpietri ${ }^{4}$ \\ Bertrand Piot ${ }^{1}$ \\ 'Department of Applied Research and \\ Development, L'Oreal Research and \\ Innovation, Paris, France; ${ }^{2}$ RB Consult, \\ Bievres, France; ${ }^{3}$ Private Dermatology \\ Consultancy Practice, Montpellier, \\ France; ${ }^{4}$ BIOTHERM International, \\ Levallois-Perret, France
}

This article was published in the following Dove Press journal:

Clinical, Cosmetic and Investigational Dermatology

25 September 2013

Number of times this article has been viewed

Objectives: Aging signs can be classified into four main categories: wrinkles/texture, lack of firmness of cutaneous tissues (ptosis), vascular disorders, and pigmentation heterogeneities. During a lifetime, skin will change in appearance and structure not only because of chronological and intrinsic processes but also due to several external factors such as gravity, sun and ultraviolet exposure, and high levels of pollution; or lifestyle factors that have important and obvious effects on skin aging, such as diet, tobacco, illness, or stress. The effect of these external factors leads to progressive degradations of tegument that appear with different kinetics. The aim of this study was to clinically quantify the effect of sun exposure on facial aging in terms of the appearance of new specific signs or in terms of increasing the classical signs of aging.

Materials and methods: This study was carried out on 298 Caucasian women from 30 years to 78 years old. The participants were divided into two groups according to their sun exposure history: 157 women were characterized as sun-seeking, and the other 141 were classified as sun-phobic. This division was made possible by dermatologist grading of heliodermal status on the basis of several observations of classic criteria: wrinkles, sagging, pigmentation heterogeneities, vascular disorders, elastosis, and so on. This work was an opportunity to complete clinical photographic tools by adding in our portfolio new scales for signs observed in the two groups. Thus, 22 clinical parameters were investigated by a panel of twelve trained experts to characterize each woman's face regarding standardized photographic scales, and thus describe the aging process.

Results: By calculating statistical correlations between the four clinical clusters (wrinkles/ texture, ptosis, vascular disorders, and pigmentation disorders), and real age and apparent age on the one hand and heliodermal status on the other hand, we identified a link between each clinical cluster and aging and the photoaging process. By comparing evaluations of clinical signs between the two groups for each 10-year cluster, we demonstrated that whatever the age, a prevalence of pigmentation disorders for the sun-seeking group (ie, pigmentation) is strongly linked to ultraviolet (UV) exposure. Meanwhile, clinical signs of ptosis are linked more to chronological aging and do not present differences between the two groups, nor, therefore, photoaging. Wrinkles and texture are affected by the two aging processes. Finally, clinical signs of vascular disorders present no evolution with age.

Conclusion: Clinical signs of aging are essentially influenced by extrinsic factors, especially sun exposure. Indeed UV exposure seems to be responsible for $80 \%$ of visible facial aging signs.

Keywords: photoaging, clinical evaluation, wrinkles, ptosis, pigmentation, UV

Correspondence: Frederic Flament Department of Applied Research and Development, L'Oreal Research and Innovation, I88, Rue Paul Hochart, 94550 Chevilly-Larue, Paris, France Email fflament@rd.loreal.com

\section{Introduction}

Distinction of faces according to sex or age, is a skill we all present with very early in our childhood. It is mainly by learning and comparing that we develop this ability. 
For clinical and biophysical researchers to understand what elements are taken into account in a given country or cultural environment when one human being meets another and assigns an age, is crucial.

Obviously, many clues allow us to estimate the age of an individual; size, posture, voice intonation, and clothes are some of the parameters taken into account. However, facial features are the most essential element because they provide enough information to evaluate appearance and so recognize who the person is in front of you. Facial features reflect not only our sex or age, but also our identity. The structure of bones and the movement of underlying muscles create facial features. Finally, skin is the exterior envelope that molds all these structures together, and thus translates our experiences. The appearance of skin reveals many life events: physiognomy and expressions are inherent to our character or education; diet or illness affects facial volumes in particular; the continuing effect of gravity pulls skin down; our behavior regarding ultraviolet (UV) exposure degrades skin quality; and finally, our lifestyle choices, such as smoking, may be responsible for premature aging by generating noxious free radicals. Facial aging is the result of several concomitant processes.

The face is constantly exposed to sunlight and gravity and presents a particularity contrary to other body sites. The face is rich in muscles that allow us, among other things, verbal and nonverbal communication through facial expressions, both static and dynamic. In addition, it is the prevalent area for visible vascular changes such as discreet erythema, vasoconstriction (pallor), or flushing (redness and sweating). By listing all these causes, it becomes clear that distinguishing and discriminating their exact effects in facial aging could be complex. Indeed, each cause will induce completely different reactions and consequences.

The literature contains plenty of articles describing clearly and accurately the effect of sunlight exposure on skin structures and the different processes of recovery after sun damage. ${ }^{1-7}$ From birth, the face is constantly exposed to sunlight, and so year by year, the skin will accumulate damage that gradually induces the appearance of visible signs of aging by marking areas of the skin, or perpetuating facial expressions. Continuous UV exposure will also lead to other changes falling under the description of photoinduced damages, such as loss of pigmentation and vascular homogeneities, loss of skin elasticity, and degradation of skin texture (elastosis, hyperkeratosis, and yellowing). The effect of sun exposure has been well described, noticeably in pathological dimension, and the necessity of photo-protection has been clearly demonstrated in the past to avoid any skin diseases like skin carcinoma. Observing and quantifying healthy facial skin in its clinical aging fluctuations with different UV exposure behavior remains less documented and an important field for investigation.

The question that arises is: how can we understand, and measure specifically, the clinical effect of sun exposure (photoaging) in facial aging relative to that of chronological aging? Several authors have estimated that this ratio could be very important, ${ }^{8}$ up to $80 \%$ of sun impact for a large part, ${ }^{9,10}$ and some publications have discussed a ratio closer to $90 \%{ }^{11}$ Can we quantify this effect? What is its contribution to facial aging? This study examines these questions. Arriving at an answer is quite difficult because chronological aging and photoaging are linked through time. In fact, the older we are, the more our face has been exposed to the sun, whatever our lifestyles and experiences. Thus, there is no control population: everyone has spent time in the sun in their lifetime, so no one can serve as an "unexposed" sample. With this study, we attempt to quantify the clinical proportion of photoaging and chronological aging in facial evolution.

\section{Materials and methods}

The study was conducted in Montpellier $\left(43^{\circ} \mathrm{N} ; 3^{\circ} \mathrm{E}\right)$. This town is located in the South of France and presents a high sun exposure level (ie, it has more than 110 days of sunshine every year).

We enrolled 298 healthy Caucasian women, aged from 30 years to 78 years, and divided them into two groups: sunseeking (S-S, 157 women) and sun-phobic (S-P, 141 women). The women presented different kinds of skin type (dry, oily, and combination) and were well-balanced across phototypes I to IV, according to Fitzpatrick classification. ${ }^{12}$ For the following assessments and results, we regrouped the volunteers into 10-year age clusters.

Two groups were established after clinical examination performed by an experienced dermatologist and after evaluation of sun behavior history by questionnaire (Sun Behavior Score history [SBSH]). A score between 0 and 3 is given for each 10-year cluster for different items: residence location, occupation, passive UV exposure, active UV exposure, and photo-protection habits. The value from 0 (none) to 3 (very) is given by considering the importance of UV exposure for the considered item. SBSH is the sum of scores for all the items and varied from 4 to 30 for volunteers in their twenties, and from 14 to 105 for volunteers in their seventies. Linked with age and phototype, SBSH is a key descriptor of the UV exposure level of each panelist. Therefore, the description 
of panel and labeling of S-S and S-P groups was performed with the following thresholds: 25 for the cluster aged 30 to 39 years, 34 for the cluster aged 40 to 49 years, 43 for the cluster aged 50 to 59 years, 51 for the cluster aged 60 to 69 years, and 60 for the cluster aged 70 to 78 years.

After observation, the dermatologist assessed heliodermal status by taking into account the classical clinical signs described in Table 1. Five grades of photo-damage were determined in this process, ranging from 0 to $4(0$, none; 1, minor; 2 , moderate; 3 , important; and 4 , major). To add reliability and accuracy, clinical evaluation was performed using two sets of standardized and validated photographic scales. First, the dermatologist used the original Skin Aging Atlas. Volume 1, Caucasian Type ${ }^{13}$ (Table 2), and second, new clinical scales specific for populations that have been more affected by UV exposure were built with the study pictures. Indeed, we took advantage of this opportunity to undergo exactly the same process of construction and validation our previously described clinical toolboxes presented in the Skin Aging Atlas. Volume 2, Asian Type ${ }^{14}$ with new standardized photographic scales to observe and quantify various clinical signs of aging and photoaging. The list of these new signs is presented in Tables 3-5. Figures 1 and 2 show examples of these new photographic scales for wrinkles/texture and pigmentation issues, which allow evaluation according to the definitions.

To make the creation of new standardized clinical scales possible, we have taken 3 facial pictures of each volunteer: both a front, full-face image and a $45^{\circ}$ image from each side. All these pictures were observed by a panel of five experts who defined new signs and their facial position and picked the most representative images for each of the considered items to obtain a linear photographic scale from grade 0 (no visible sign manifestation) to a maximum grade found in the population. Validation of criteria relevance and linearity of the scales is a prerequisite

Table I Clinical signs used by dermatologists to establish heliodermal status

\begin{tabular}{lll}
\hline Signs & Severity & Scale \\
\hline Pigmentation & Absence, clods, puddles, poikiloderma & 0 to 3 \\
Depigmentation & Absence, drops, plates & 0 to 3 \\
Pachydermic appearance & Absence, presence & 0,1 \\
Elastosis & None, mild, moderate, severe & 0 to 3 \\
Cutaneous atrophy & None, mild, moderate, severe & 0 to 3 \\
Vascular disorders & None, mild, moderate, severe & 0 to 3 \\
Fine lines & None, mild, moderate, severe & 0 to 3 \\
Wrinkles & None, mild, moderate, severe & 0 to 3 \\
Ptosis & None, cheeks, eyelid, face & 0 to 3 \\
\hline
\end{tabular}

Table 2 Clinical aging signs of the face, described by atlases, and correlations with age and heliodermal status

\begin{tabular}{lll}
\hline Type and signs & Age & Heliodermal status \\
\hline Wrinkles* & & \\
$\quad$ Forehead wrinkles* & 0.335 & 0.282 \\
Crow's foot wrinkles* & 0.602 & 0.478 \\
Glabellar wrinkles* & 0.553 & 0.396 \\
Underneath eye wrinkles* & 0.546 & 0.395 \\
Upper-lip wrinkles* & 0.558 & 0.531 \\
Corner of the mouth* & 0.520 & 0.402 \\
Ptosis & & \\
Eye bag & 0.529 & 0.303 \\
Lower face ptosis & 0.764 & 0.498 \\
\hline
\end{tabular}

Note: *Signs most affected by UV exposure.

Abbreviation: UV, ultraviolet.

to ensuring that evaluation will be scientifically correct. We obtained these data by implementing a paired tests approach, as discussed in the Skin Aging Atlas. Volume 3, Afro-American Type ${ }^{15}$ and other literature, ${ }^{16,17}$ which consists of a classification by 15 volunteers on a screen in standardized conditions of lighting, position, and calibration of each grade for a considered scale by comparing all the possible pairs of two grades. With this step, we have been able to establish which grades are significantly different than others, and thus had the opportunity to remove, adjust, or change pictures, which ensured the absence of discrimination.

By merging the two sets of clinical scales, we obtained a complete mapping of facial aging covering four major axes: wrinkles and relief texture (Table 3), lack of firmness, pigmentary disorders (Table 4), and vascular alterations (Table 5). The description of new relevant signs, and examples of more severe pictures regarding these new clinical scales for this classification, are presented in Table 3.

In addition to the dermatologist evaluation and to characterize our panel of 298 women completely on all the 22 selected scales, we used a group of 12 experts trained to make objective evaluations regarding each standardized clinical scale (reproducibility and repeatability). Evaluation was carried out on pictures resized and reframed according to a standard operational procedure with editing software (Photoshop $^{\circledR}$, version 10; Adobe Systems Incorporated, New York, NY, USA) to ensure that only the scored facial area was displayed on the screen. Pictures are presented to each expert in random order to eliminate any possibility of bias. The evaluation took place in entirely standardized conditions of lighting, position (expert is seated $1 \mathrm{~m}$ from the screen), and calibration (a 24-inch, $1,920 \times 1,200$-pixel high-resolution screen calibrated with 
Table 3 Wrinkles/texture signs and correlations with age and heliodermal status

\begin{tabular}{|c|c|c|c|}
\hline Signs & Heliodermal status & Age & Wrinkles and texture \\
\hline $\begin{array}{l}\text { Upper-lip texture* } \\
\text { In addition to more or less deep vertical lines, } \\
\text { the upper lip has a thick aspect, is padded, } \\
\text { and has a pronounced microrelief forming a grid }\end{array}$ & $0.54 I$ & 0.579 & \\
\hline $\begin{array}{l}\text { Cheek folds* } \\
\text { Deep folds completely anarchic and presenting } \\
\text { no specific directions link to muscular } \\
\text { movements of face }\end{array}$ & 0.515 & 0.573 & \\
\hline $\begin{array}{l}\text { Lower lip wrinkles* } \\
\text { Wrinkles more or less deep from corner } \\
\text { of lower lip and extending downward from } \\
\text { the chin }\end{array}$ & 0.495 & 0.531 & \\
\hline $\begin{array}{l}\text { Jawline folds* } \\
\text { Folds begin at the ear lobe created by a thick skin, } \\
\text { marked by dryness and elastosis, and extending } \\
\text { over the jaw and neck giving a crumpled paper } \\
\text { appearance }\end{array}$ & 0.437 & $0.58 \mathrm{I}$ & \\
\hline $\begin{array}{l}\text { Wrinkles created by lower face ptosis* } \\
\text { A more or less deep fold is created at the zonal } \\
\text { level of separation of chin and cheek }\end{array}$ & 0.495 & 0.523 & \\
\hline $\begin{array}{l}\text { Chin texture* } \\
\text { In addition to dimples, the area of the chin and cheek } \\
\text { areas adjacent have a thick skin appearance, } \\
\text { are padded, and have a pronounced microrelief } \\
\text { forming a grid }\end{array}$ & 0.564 & 0.623 & \\
\hline
\end{tabular}

Notes: Correlations are with age and heliodermal status. *Signs most affected by UV exposure.

Abbreviation: UV, ultraviolet.

a colorimeter). We end the process by taking the average score of all the panelists and obtaining clinical assessment for each chosen sign for each volunteer. This approach has allowed us to guarantee the objectivity and relevance of the evaluation with complete randomization and no impact of face or any environmental issues. To ensure robustness of this process, several pictures were presented twice during evaluations.

Finally, we added to our investigations a quiz phase by asking 30 naïve Caucasian panelists (between 18 to 60 years of age without any specification on marital status or profession) their opinion regarding the full-face picture of the study volunteers. The purpose of this step is to record the apparent age perceived by looking at a photograph. The question asked was: "what age do you think this woman is?"

\section{Statistical analysis}

Analysis of variance at one factor, followed by a Tukey comparison test, was used to investigate the link between age and heliodermal status, as well as between phototype and heliodermal status. The effects of age and UV exposure were characterized by a sum of clinical criteria divided into four clinical clusters (wrinkles, sagging, 
Table 4 Pigmentation disorders and correlations with age and heliodermal status

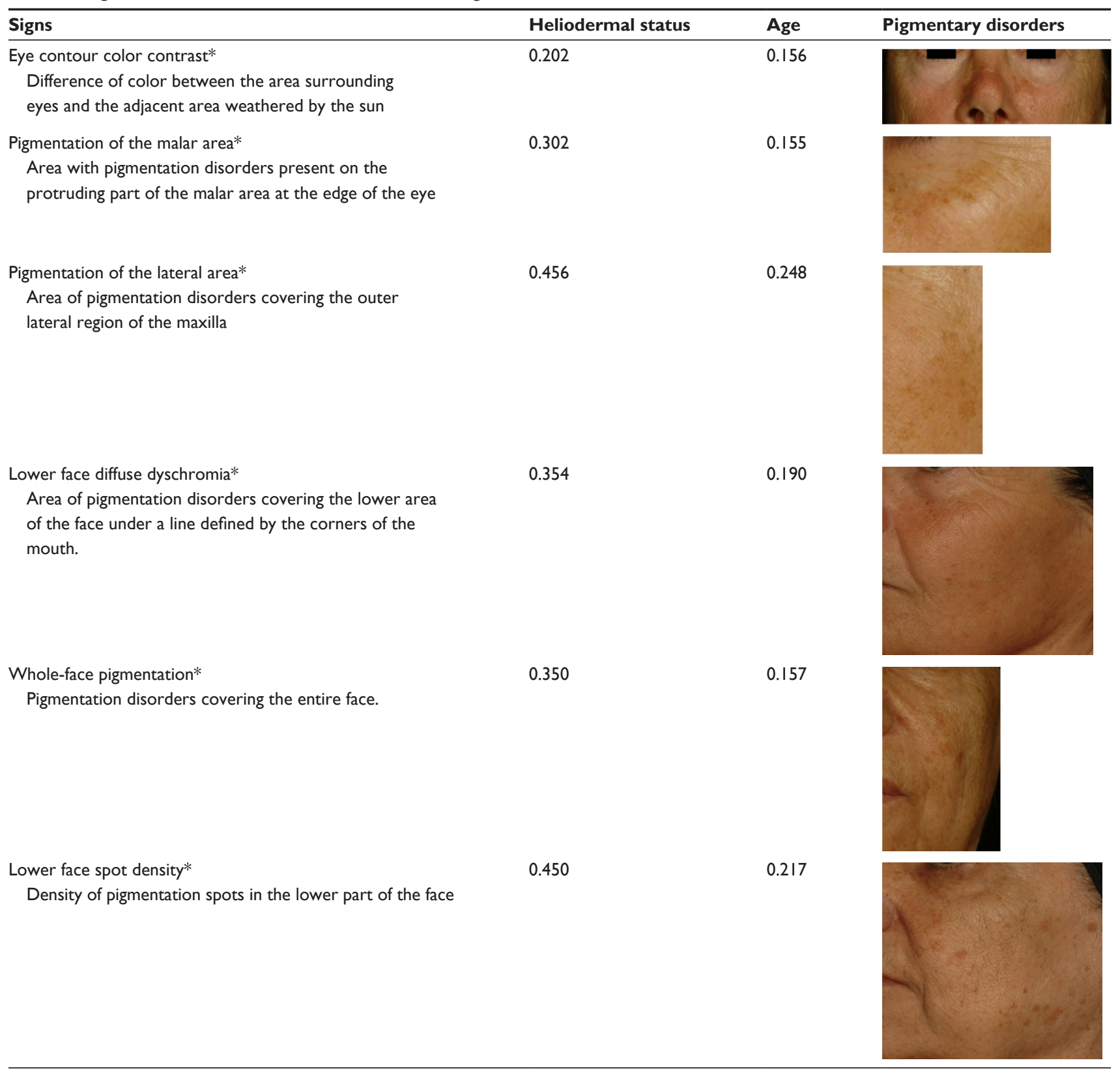

Note: *Signs most affected by UV exposure. Abbreviation: UV, ultraviolet.

pigmentary disorders, and vascular alterations), defined in Tables 2-5.

To determine the influence of UV effect on clinical signs regarding evolution essentially resulting from age, Pearson coefficients were computed between these four clinical clusters and heliodermal status, as well as between the clinical clusters and the real age of each volunteer. Finally, the correlation with apparent age was also calculated to ensure the most relevant clinical signs were taken into account in age perception and to be able to conclude what the influence of photoaging is on our appearance. To avoid any bias, all the values coming from scales presenting different ranges were normalized to five.

A $t$-test for independent sample was used to compare groups in each 10-year cluster for S-S and S-P panels regarding each clinical cluster. A similar statistical approach was used to compare the difference between apparent age and real age. 
Table 5 Microvascular disorders and correlations with age and heliodermal status

\begin{tabular}{llll}
\hline Signs & Heliodermal status & Age & Microvascular signs \\
\hline $\begin{array}{l}\text { Couperosis/rosacea } \\
\text { Microvascular alterations on the cheekbone area }\end{array}$ & 0.257 & 0.086 \\
\hline $\begin{array}{l}\text { Vascular disorders } \\
\text { on the face }\end{array}$ & & & \\
& 0.140 & 0.031 & \\
\hline
\end{tabular}

Note: The signs presented here are not related to UV exposure.

Abbreviation: UV, ultraviolet.

\section{Results}

In Figure 3, we show the evolution of heliodermal status with age. First, we can observe that the effect of UV exposure increases with age. All age clusters are statistically different from the younger volunteers except for the cluster of those aged 40 years to 49 years, for which the difference is only significant at $10 \%$.

We have computed correlations between real age and heliodermal status (0.499) and apparent age and heliodermal status (0.606). At the same time, we observed that the correlation between apparent and real

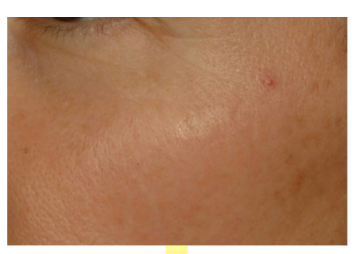

Grade 0

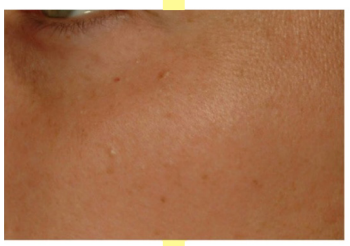

Grade 1

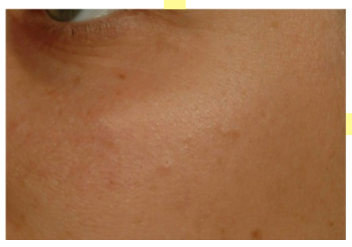

Grade 2

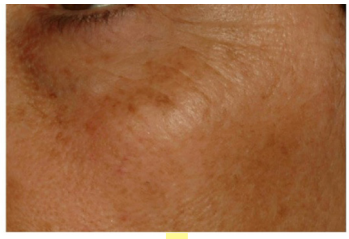

Grade 5

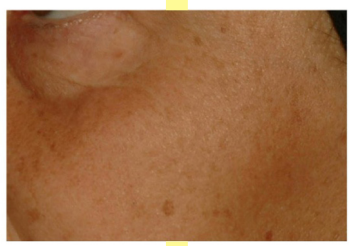

Grade 4

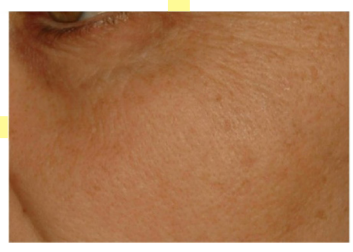

Grade 3
Figure I Clinical standardized photographic scale of pigmentation of malar area. age could be approached at 0.904 . We also noticed that heliodermal status is more correlated to apparent age than real age. Clinical signs of photoaging seem more important both in our appearance and in its perception by other people.

Correlations between the four clinical clusters and real age or apparent age on one hand, and heliodermal status on the other, are summed up in Table 6. The classification is presented according to its correlation with apparent age, from the highest to the lowest. This ranking shows us that pigmentation-related clinical parameters are the most linked to photoaging but are not the most important related to age. In contrast, wrinkles are strongly bound to age and heliodermal status. Sagging manifestations seem to be more linked to chronological aging. Finally, vascular disorders do not present a high correlation with chronological age or heliodermal status.

Figures 4-7 show the difference for each 10-year cluster between the S-S and S-P groups created in terms of UV exposure behavior. On the basis of these data (Figures 4 and 6) and previous correlations, we demonstrate our conclusions: whatever the age, a prevalence of pigmentation disorders occurs, with significant differences between the two groups, and these signs are the most connected to photoag-

Table 6 Correlations between clinical aging signs and age and heliodermal signs

\begin{tabular}{llll}
\hline & $\begin{array}{l}\text { Apparent } \\
\text { age }\end{array}$ & $\begin{array}{l}\text { Real } \\
\text { age }\end{array}$ & $\begin{array}{l}\text { Heliodermal } \\
\text { status }\end{array}$ \\
\hline Wrinkles/texture & $0.860^{*}$ & $0.740^{*}$ & $0.604^{*}$ \\
Ptosis & $0.803^{*}$ & $0.774^{*}$ & $0.552^{*}$ \\
Pigmentation disorders & $0.416^{*}$ & $0.317^{*}$ & $0.632^{*}$ \\
Vascular disorders & 0.054 & 0.000 & 0.181 \\
\hline
\end{tabular}

Note: *Significant at $0.5 \%$. 


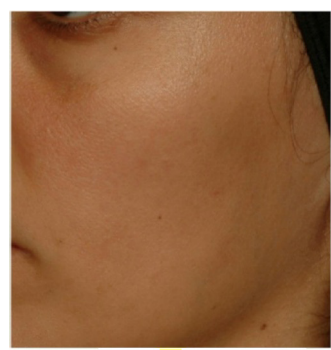

Grade 0

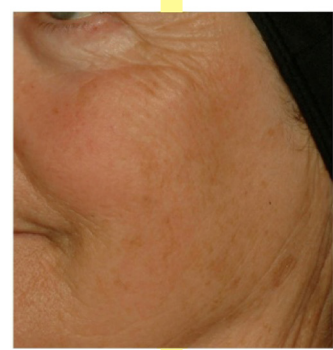

Grade 1

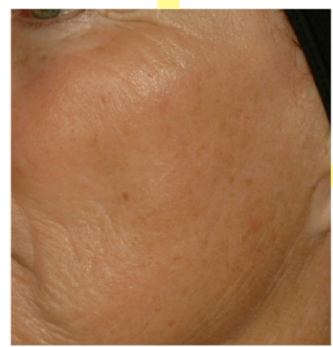

Grade 2

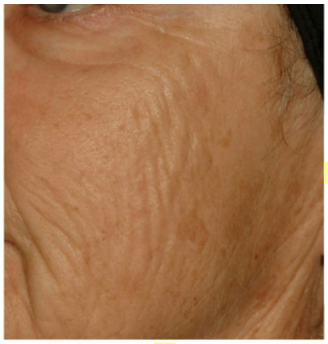

Grade 5

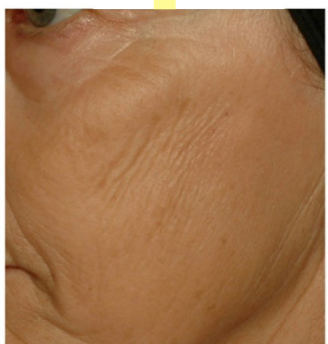

Grade 4

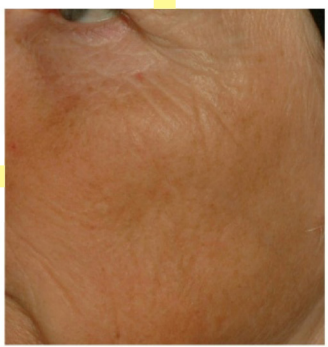

Grade 3

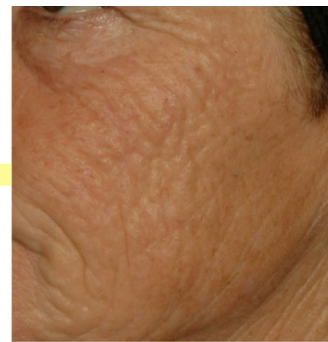

Grade 6

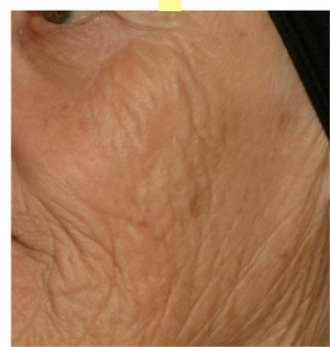

Grade 7

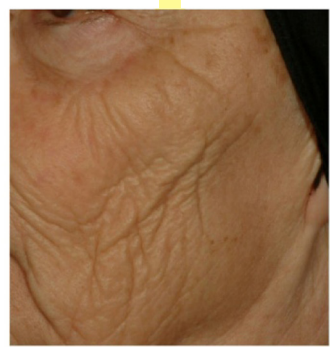

Grade 8

Figure 2 Clinical standardized photographic scale of cheek folds.

ing. Concerning wrinkles and skin texture quality, significant differences between the two groups appear after age 50 years. No statistical differences for the signs of sagging are observed between the S-S and S-P groups (Figure 5). The amplitude of variations for vascular signs (Figure 7) is not very high, and an evolution with age has not been established; however, there is a significant difference in the fifties, with a maximum for this parameter.

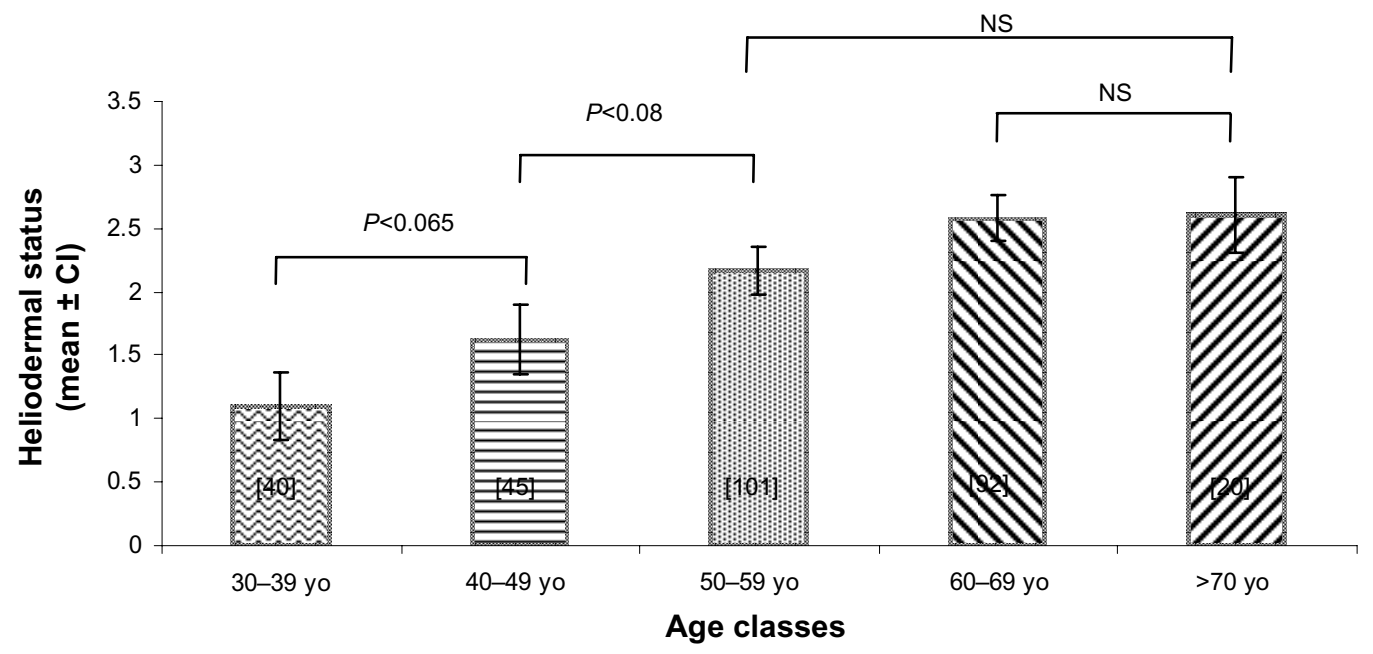

Figure 3 Heliodermal status grade (mean \pm confidence interval) in each age cluster. All bars are significantly different from the other, with an exception being the comparison marked on the graph. The number of people in each cluster is indicated between the brackets.

Abbreviations: yo, years, $\mathrm{Cl}$, confidence interval; NS, non significant. 


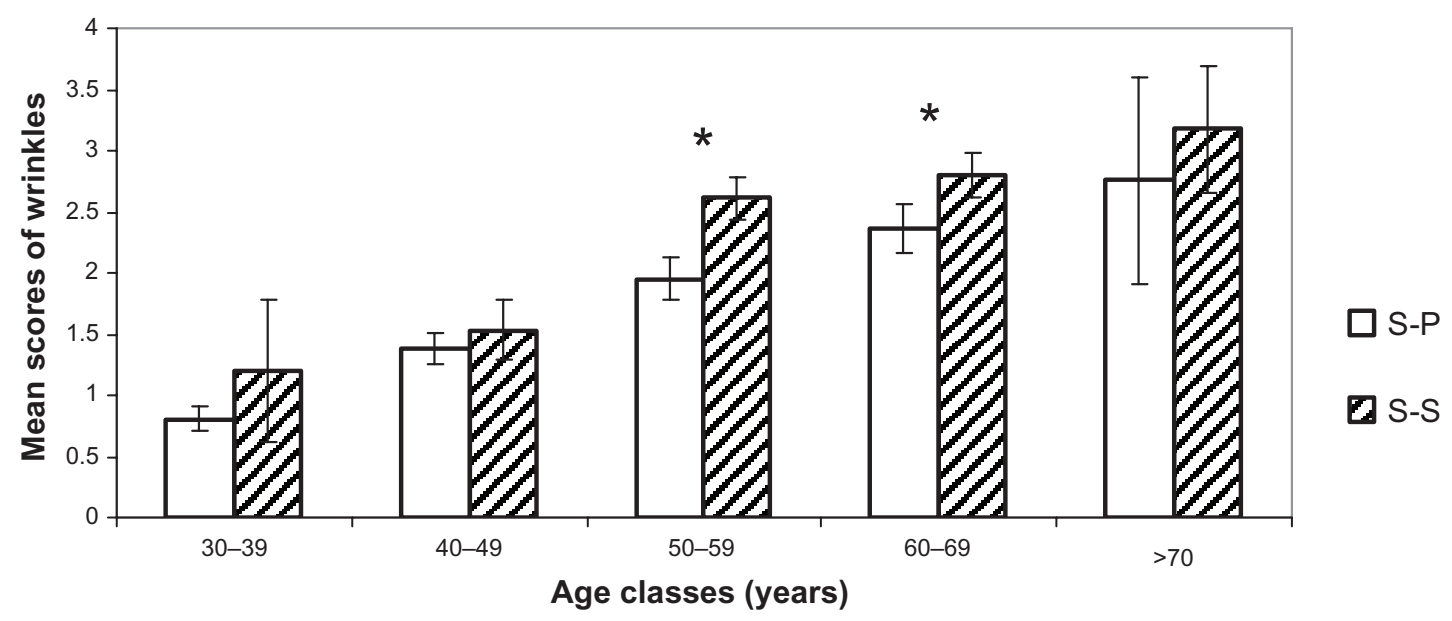

Figure 4 Comparison of wrinkles and relief texture. Mean scores ( $\pm \mathrm{Cl} 95 \%)$ for each age cluster between S-S and S-P. Note: *Statistically significant difference.

Abbreviations: S-P, sun-phobic; S-S, sun-seeking; Cl, confidence interval.

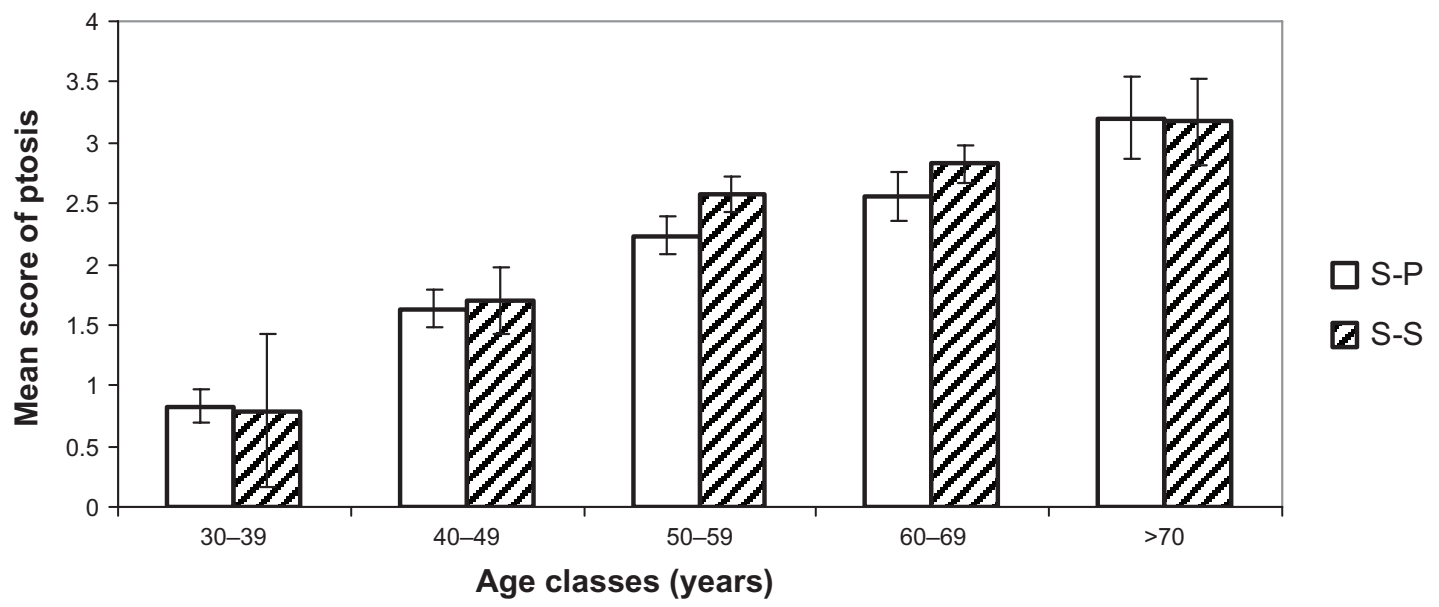

Figure 5 Comparison of ptosis and sagging. Mean scores $( \pm \mathrm{Cl} 95 \%)$ for each age cluster between S-S and S-P.

Note: There is no statistically significant difference between S-P and S-S groups.

Abbreviations: S-P, sun-phobic; S-S, sun-seeking; Cl, confidence interval.

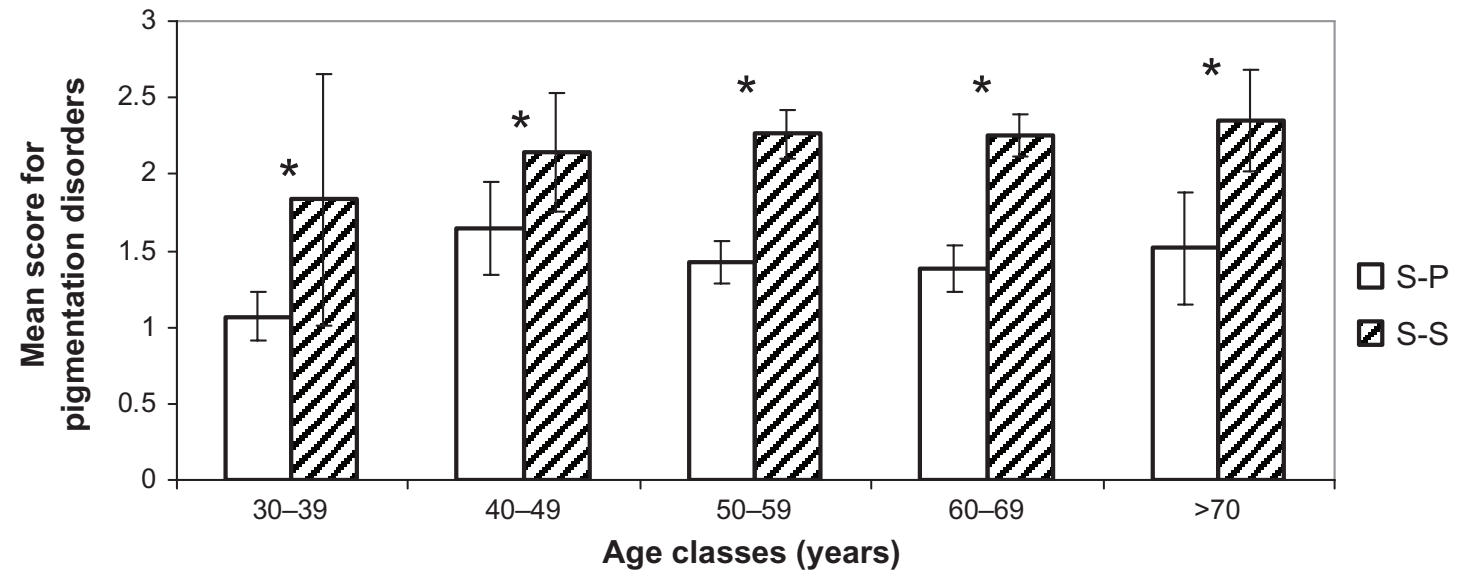

Figure 6 Comparison of pigmentation disorders. Mean scores ( $\pm \mathrm{Cl}$ 95\%) for each age cluster between S-S and S-P. Note: *Statistically significant difference.

Abbreviations: S-P, sun-phobic; S-S, sun-seeking; CI, confidence interval. 


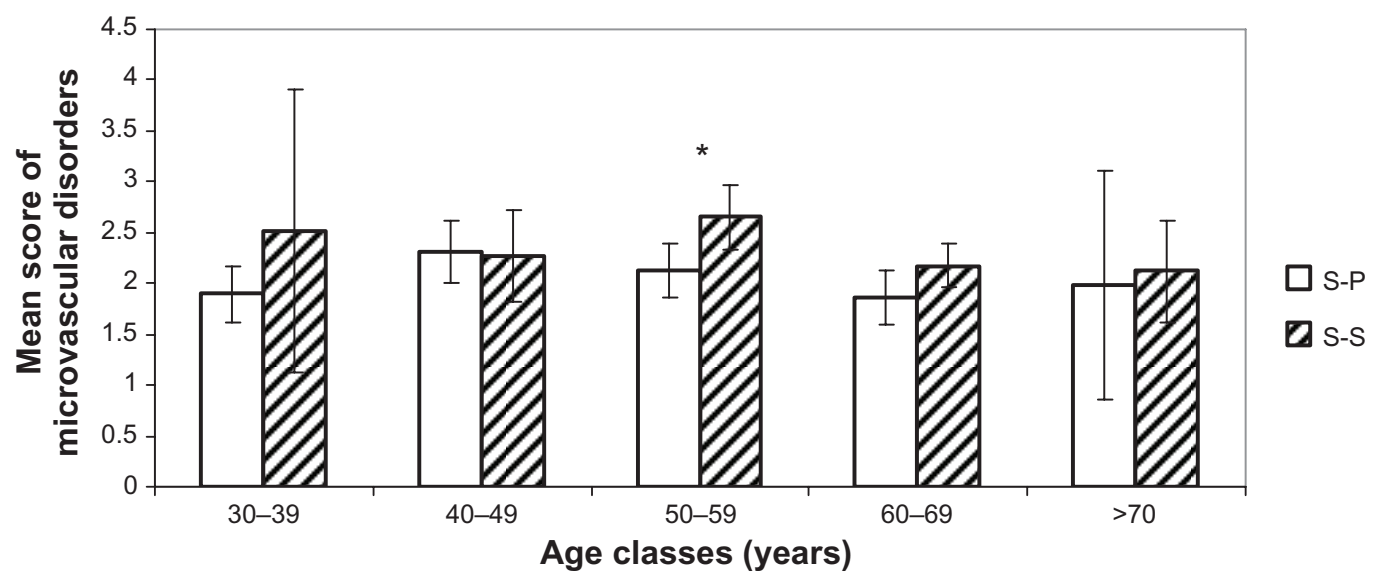

Figure 7 Comparison of microvascular disorders. Mean scores $( \pm \mathrm{Cl} 95 \%)$ for each age cluster between S-S and S-P.

Note: *Statistically significant difference.

Abbreviations: S-P, sun-phobic; S-S, sun-seeking; $\mathrm{Cl}$, confidence interval.

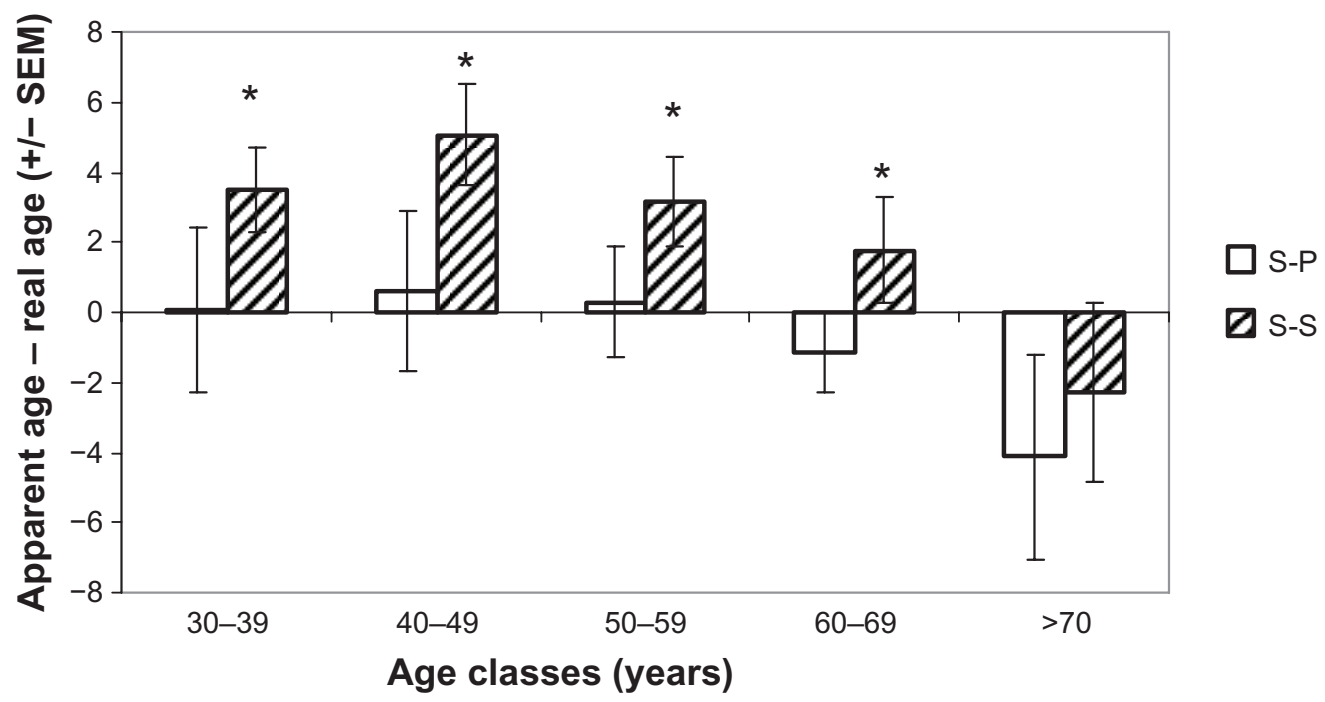

Figure 8 Difference between apparent and chronological age for the S-S and S-P groups.

Notes: *Statistically significant difference between bars. A positive difference means that the person looks older than their age.

Abbreviations: S-P, sun-phobic; S-S, sun-seeking; SEM, standard error of the mean.

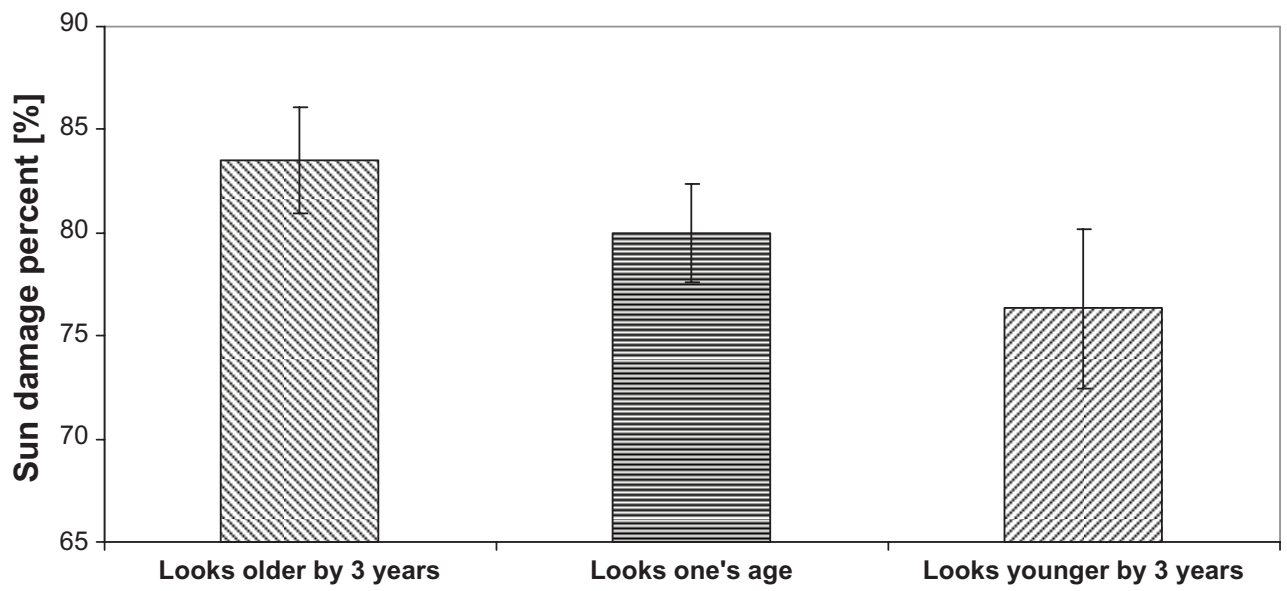

Figure 9 Percentage of sun damage is predictive of how old a woman looks. 
The study of the effect of UV exposure on our appearance is summarized in Figure 8. For all the women in the S-S and S-P groups, we analyzed on all age clusters the difference between apparent age, as estimated by a panel of 30 people, and real age. With the exception of the eldest cluster, we observed significant differences between the two populations (ie, S-S volunteers looked older than their real age). This difference seems to decrease over time. We also observed for the elder group (older than 70 years) that people look younger than their real age with no significant difference in sun exposure.

Finally, to quantify the effect of sun exposure on facial aging, we chose the following method: on the basis of the quantification of each volunteer, by way of all the photographic scales presented earlier, a sum was done of all signs most affected by UV exposure (the 18 parameters marked with an asterisk in Tables 2-5), which was then compared with the sum of all clinical signs established for facial aging (22 parameters). We are able to determine a new ratio, sun damage percentage (SDP), which represents the percentage between specific photoaging signs and clinical signs. By computing this SDP, we could assess the effect of sun exposure on the face. On average, the parameter is $80.3 \% \pm 4.82 \%$.

Differences between the real and apparent ages could be divided into three groups (Figure 9): the women who seem visually older by more than 3 years compared with their real age, those who look their age, and finally, women who seem younger by more than 3 years compared with their real age. At a threshold of $80 \%$ for the SDP, women have similar apparent age to real age. If SDP increases (82\%), then apparent age becomes higher than real age, and this woman looks older. Conversely, a decrease of SDP (78\%) means that the woman looks younger.

\section{Discussion}

To the best of our knowledge, at this time there are no studies that provide an objective quantification of the effect of sun exposure on facial aging. This article attempts to provide some elements for the quantification and understanding of the effect of UV sun exposure on skin. Of course, as described earlier, separating concomitant phenomena involved in both chronological aging and photoaging is a very complex process.

Sun exposure is essential in occidental culture and is important not only for mood and emotional well-being but also at a physiological level, especially for synthesis of vitamin D. For the fair-skinned population, the effect of sun is more severe because of the body's natural defenses being less efficient than in those with darker skins. In this study, by using a simple criterion summarizing attitudes to the sun, it was possible to define two groups of women whose sun behavior stories were different. A comparison between these two groups allowed us to clearly demonstrate the effect of UV exposure in skin aging. Pigmentation signs are most visible and most specific of these effects. This aspect has already been described and explained very often in the literature. . $, 8,18,19$

In this study, we attempted to describe in the most simple and complete way all pigmentary heterogeneities of the face. This description is intended to define not only a way to characterize precisely all the facial areas covered but also the intensity and distribution of pigmentation disorders in each of these areas. It is the clinical observation of all the pictures that led us to the creation of standardized photographic scales. Quantification by a panel of trained experts is a pathway for great objectivity because each sign is evaluated independent of all the other signs, because we could observe only one sign on the screen. The fact that it was not possible to be influenced by other areas and other signs, and that the result is the average of the twelve experts, ensured the reliability of the observations. The use of clinical scales as defined in this article enables us to statistically characterize and detect even small variations, for example, when they first appear (Figures 4-7).

Clearly, clinical signs of pigmentation are strongly determined by UV exposure. It is a more complicated matter to conclude and separate chronological and sun-induced effects on wrinkles and skin texture ${ }^{20-23}$ We observe that the clinical signs describing wrinkles and skin texture are correlated in a very similar way in both age and heliodermal status. According to Griffiths, ${ }^{18}$ photoaging is essentially characterized by "coarse and fine wrinkles," whereas signs of chronological aging can be described as a skin with fine wrinkles. For Griffiths, coarse wrinkles are pathognomonic signs of extrinsic aging, although they are not only caused by sun damage. Wrinkle characteristics of intrinsic aging also could present themselves as "fine lines." This confirms the great difficulty in differentiating this parameter for chronological and sun-exposure effects. The photographic clinical scales used during this study (Tables 2 and 3) could be considered, with grade 1 as "coarse wrinkles," and therefore completely included in photoaging signs. ${ }^{18}$

Signs related to sagging of the tissues are not considered in this study as being linked to the sun. Indeed, the correlation of this clinical cluster is more important with chronological age than heliodermal status. Conversely, we have demon- 
strated that there are no significant differences between S-S and S-P groups, whatever the age. The remaining positive correlation with heliodermal status could be explained by the fact that these criteria are assessed by the dermatologist indoors. This assertion is confirmed by literature, ${ }^{7,18}$ which does not mention sagging as an extrinsic factor. The sagging we evaluate with accurate photographic scales is more linked to Earth's gravity.

Vascular disorders are not correlated with either age or photo-damage status. In Figure 7, we observe that this phenomenon is opposite that of other clinical clusters. However, literature often quotes telangiectasias ${ }^{7,18,21}$ as important signs of sun damage. The interpretation could be that an increase in vascular signs resulting from subclinical inflammation gradually decreases during intrinsic aging , $^{722}$ and leads to a depletion of cutaneous blood vessels. At the same time, there is a thickening of the epidermis and stratum corneum, which makes the vascular network less visible. This could explain biphasic kinetics slightly passing through a maximum around age of 50 years, where the difference between S-S and S-P groups becomes significant.

Finally, we show that photoaged women look older than women who protect themselves from the sun. This factor is higher when you are younger. Pigmentation and wrinkles/texture, especially wrinkles around lips, are signs that make a person look older earlier. It is an important observation from this study that the signs of photoaging influence age appreciation through the eyes of other people. An increase or decrease of $2 \%$ of the SDP can change the apparent age by plus or minus 3 years. Thus, the term "premature skin" could correspond very well to description of photoaged skin, which is actually prematurely aged skin.

\section{Conclusion}

With all the elements described in this study, we could calculate the importance of UV and sun exposure in the visible aging of a Caucasian woman's face. This effect is about $80 \%$.

The interactions between chronological and photoinduced aging are complex, and the quantification of only the effect of sun-exposure is difficult to obtain. Our approach of using new descriptive skin-aging atlases is a solution to specify the extrinsic influence. Twenty-two clinical signs are used to describe and assess facial aging, wrinkles and skin texture, sagging of tissues, pigmentation manifestations, and vascular disorders. This study seems to confirm that pigmentation heterogeneity is a pure photoaging sign, whereas sagging of tissues is essentially a result of chronological aging. Vascular disorders could be considered as a precursor of future photoaging. Wrinkles and skin texture are influenced by both extrinsic and intrinsic aging, depending on the behavior of the individual with regard to the sun. The study confirms the accountability of sun exposure in premature aging of the face.

\section{Disclosure}

The authors report no conflicts of interest in this work.

\section{References}

1. Lavker RM, Gerberick GF, Veres D, Irwin CJ, Kaidbey KH. Cumulative effects from repeated exposures to suberythemal doses of UVB and UVA in human skin. J Am Acad Dermatol. 1995;32(1):53-62.

2. Lavker RM. Cutaneous aging: chronologic versus photoaging. In: Gilchrest BA, editor. Photodammage. Cambridge, UK: Blackwell Science; 1995:123-135.

3. El-Domyati M, Attia S, Saleh F, et al. Intrinsic aging vs photoaging: a comparative histopathological, immunohistochemical, and ultrastructural study of skin. Exp Dermatol. 2002;11(5):398-405.

4. Giacomoni PU, Nadaud JF, Straface E, Donelli G, Heenen M, Malorni W. Morphological alterations and cell blebbing in UV-irradiated human epidermis. Arch Dermatol Res. 1998;290(3): 163-166.

5. Baumann L. Skin ageing and its treatment. J Pathol. 2007;211(2): 241-251.

6. Yaar M, Gilchrest BA. Photoageing: mechanism, prevention and therapy. Br J Dermatol. 2007;157(5):874-887.

7. Gilchrest BA. Skin aging and photoaging: an overview. J Am Acad Dermatol. 1989;21(3 Pt 2):610-613.

8. Uitto J. Understanding premature skin aging. N Engl J Med. 1997; 337(20):1463-1465.

9. Langton AK, Sherratt MJ, Griffiths CE, Watson RE. A new wrinkle on old skin: the role of elastic fibres in skin ageing. Int J Cosmet Sci. 2010;21:1-2.

10. Farage MA, Miller KW, Elsner P, Maibach HI. Intrinsic and extrinsic factors in skin ageing: a review. Int J Cosmet Sci. 2008;30(2):87-95.

11. Südel KM, Venzke K, Mielke H, et al. Novel aspects of intrinsic and extrinsic aging of human skin: beneficial effects of soy extract. Photochem Photobiol. 2005;81(3):581-587.

12. Fitzpatrick TB. The validity and particularity of sun-reactive skin types I through VI. Arch Dermatol. 1988;124(6):869-871.

13. Bazin R, Doublet E. Skin Aging Atlas. Volume 1. Caucasian Type. Paris: Editions Med'Com; 2007.

14. Bazin R, Flament F. Skin Aging Atlas. Volume 2. Asian Type. Paris: Editions Med'Com; 2010.

15. Bazin R, Flament F, Giron F. Skin Aging Atlas. Volume 3. Afro-American Type. Paris: Editions Med'Com, 2012.

16. David HA. The Method of Paired Comparisons. 2nd ed. London: Charles Griffin and Company, 1988.

17. Gacula MC, Singh J. Statistical Methods in Food and Consumer Research. Orlando, FL: Academic Press; 1984:410-424.

18. Griffiths CE. The clinical identification and quantification of photodamage. Br J Dermatol. 1992;127(41):37-42.

19. Glogau RG. Aesthetic and anatomic analysis of the aging skin. Semin Cutan Med Surg. 1996;15(3):134-138.

20. Kligman AM, Zheng P, Lavker RM. The anatomy and pathogenesis of wrinkles. Br J Dermatol. 1985;113(1):37-42.

21. Fitzpatrick RE, Goldman MP, Satur NM, Tope WD. Pulsed carbon dioxide laser resurfacing of photo-aged facial skin. Arch Dermatol. 1996;132(4):395-402. 
22. Alexiades-Armenakas M. Rhytides, laxity, and photoaging treated with a combination of radiofrequency, diode laser, and pulsed light and assessed with a comprehensive grading scale. J Drugs Dermatol. 2006;5(8):731-738.
23. Giacomoni PU, Rein G. Skin aging: A generalization of the microinflammatory hypothesis. In Farage MA, Miller KW, Maibach HI, editors. Textbook of Aging Skin. Berlin: Springer Verlag; 2010: 789-796.

\section{Publish your work in this journal}

Clinical, Cosmetic and Investigational Dermatology is an international, peer-reviewed, open access, online journal that focuses on the latest clinical and experimental research in all aspects of skin disease and cosmetic interventions. All areas of dermatology will be covered; contributions will be welcomed from all clinicians and basic science researchers globally. This journal is indexed on CAS

The manuscript management system is completely online and includes a very quick and fair peer-review system, which is all easy to use. Visit http://www.dovepress.com/testimonials.php to read real quotes from published authors.

Submit your manuscript here: http://www.dovepress.com/clinical-cosmetic-and-investigational-dermatology-journal 